\title{
The general configuration-space Faddeev formalism for study- ing pd scattering
}

\author{
Vladimir M Suslov ${ }^{1, a}$, Mikhail A Braun², Igor Filikhin ${ }^{1}$, Ivo Slaus ${ }^{3}$, and Branislav Vlahovic ${ }^{1}$ \\ ${ }^{1}$ North Carolina Central University, 1801 Fayetteville St., Durham, NC, USA \\ ${ }^{2}$ Dep. of High Energy physics, University of St. Petersburg, St. Petersburg, 199034 Russia \\ ${ }^{3}$ Rudjer Boskovich Institute, Zagreb, 10000 Croatia
}

\begin{abstract}
The configuration-space Faddeev equations are derived for p-d scattering taking into account the difference in interaction between the participant particles. Appropriate modifications have been made in the well-known configuration-space equations for n-d scattering. To show the effect of these modifications, the s-wave calculations are performed for bound state and scattering problems. We model the charge symmetry breaking effect for ${ }^{3} \mathrm{H}$ and ${ }^{3} \mathrm{He}$ with a modified Malfliet-Tjon MT I-III potential. Results obtained for elastic $\mathrm{n}-\mathrm{d}$ and $\mathrm{p}-\mathrm{d}$ scattering at $\mathrm{E}_{\text {lab }}=14.1 \mathrm{MeV}$ are compared with our prediction (Ref. [1]) and those of the Los-Alamos/Iowa group (Ref. [2]) .
\end{abstract}

\section{Introduction}

The isotopic formalism was developed for the study of neutron-deuteron scattering in the framework of the configuration space Faddeev equations (Ref. [3]). Charge-independence breaking in the threenucleon system was investigated in elastic neutron-deuteron and breakup prosesses (Ref. [4]). Here we study proton-deuteron scattering. Presence of the electromagnetic interaction requires one to consider the neutron and proton to be different particles and precludes literal use of the isotopic formalim of (Ref. [3]). So the FNNM equations used in (Ref. [5]) have to be changed.

Taking the neutron as particle 1 and protons as particles 2 and 3 we have the requirement $\Psi(1,2,3)=-\Psi(1,3,2)$. To satisfy this condition, we present $\Psi$ in terms of Faddeev components as

$$
\Psi(1,2,3)=\Phi_{1}(1,2,3)+\Phi_{2}(2,3,1)-\Phi_{2}(3,2,1),
$$

where it is understood that in $\Phi(i, k, l)$ particles are grouped as $i+(k l)$ and $\Phi_{1}$ is antisymmetric in the last pair of arguments: $\Phi_{1}(1,2,3)=-\Phi_{1}(1,3,2)$. As function $\Phi_{2}(2,3,1)$ has no definite properties under interchange $3 \leftrightarrow 1$ we encounter permutations which are not cyclic $P_{12}(231)=(321), P_{13}(123)=$ (321). In terms of these operators and operators $P^{ \pm}$we obtain for the independent components $\Phi_{1}$ and $\Phi_{2}$ a system

$$
\begin{aligned}
& \left(E-\Delta-v_{1}(2,3)\right) \Phi_{1}(1,2,3)=v_{1}(2,3)\left(P^{-} \Phi_{2}(1,2,3)-P_{13} \Phi_{2}(1,2,3)\right), \\
& \left(E-\Delta-v_{2}(3,1)\right) \Phi_{2}(2,3,1)=v_{2}(3,1)\left(P^{+} \Phi_{1}(2,3,1)-P_{12} \Phi_{2}(2,3,1)\right) .
\end{aligned}
$$

\footnotetext{
a e-mail: vsuslov@nccu.edu
} 
Here $v_{1}(2,3)$ is a sum of the Coulomb and nuclear potentials and $v_{2}(3,1)$ is the pure nuclear potential:

$$
v_{1}(2,3)=v_{c}(2,3)+v_{p p}(2,3), \quad v_{2}(3,1)=v_{p n}(3,1) .
$$

The final pair of equations is essentially the same as for the $n d$ case with changes described above.

$$
\begin{gathered}
{\left[E+\frac{\hbar^{2}}{m}\left(\partial_{x_{1}}^{2}+\partial_{y_{1}}^{2}\right)-v_{\alpha}^{\lambda l}\left(x_{1}, y_{1}\right)\right] \Phi_{1, \alpha}^{\lambda_{0}, s_{0}, M_{0}}\left(x_{1}, y_{1}\right)=\sum_{\beta}\left[v_{c}\left(x_{1}\right)+v_{p p}\left(x_{1}\right)\right]_{\alpha \beta}\left[\Phi_{1, \beta}^{\lambda_{0} s_{0}, M_{0}}\left(x_{1}, y_{1}\right)+\right.} \\
\frac{1}{2} \int_{-1}^{1} d u \sum_{\gamma}\left(g_{\beta \gamma}^{(-)}\left(y_{1} / x_{1}, u\right) \Phi_{2, \gamma}^{\lambda_{0}, s_{0}, M_{0}}\left(x_{2}, y_{2}\right)-g_{\beta \gamma}^{(13)}\left(y_{1} / x_{1}, u\right) \Phi_{3, \gamma}^{\lambda_{0}, s_{0}, M_{0}}\left(x_{3}, y_{3}\right)\right),
\end{gathered}
$$

where $\left(x_{2}, y_{2}\right)=P^{-}\left(x_{1}, y_{1}\right)$ and $\left(x_{3}, y_{3}\right)=P_{13}\left(x_{1}, y_{1}\right)$.

$$
\begin{gathered}
{\left[E+\frac{\hbar^{2}}{m}\left(\partial_{x_{2}}^{2}+\partial_{y_{2}}^{2}\right)-v_{\alpha}^{\lambda l}\left(x_{2}, y_{2}\right)\right] \Phi_{2, \alpha}^{\lambda_{0}, s_{0}, M_{0}}\left(x_{2}, y_{2}\right)=\sum_{\beta}\left[v_{p n}\left(x_{2}\right)\right]_{\alpha \beta}\left[\Phi_{2, \beta}^{\lambda_{0} s_{0}, M_{0}}\left(x_{2}, y_{2}\right)+\right.} \\
\frac{1}{2} \int_{1}^{1} d u \sum_{\gamma}\left(g_{\beta \gamma}^{(+)}\left(y_{2} / x_{2}, u\right) \Phi_{1, \gamma}^{\lambda_{0}, s_{0}, M_{0}}\left(x_{1}, y_{1}\right)-g_{\beta \gamma}^{(12)}\left(y_{2} / x_{2}, u\right) \Phi_{3, \gamma}^{\lambda_{0}, s_{0}, M_{0}}\left(x_{3} y_{3}\right)\right),
\end{gathered}
$$

where $\left(x_{1}, y_{1}\right)=P^{+}\left(x_{2}, y_{2}\right)$ and $\left(x_{3}, y_{3}\right)=P_{12}\left(x_{2}, y_{2}\right)$. In these formulas the multi index $\alpha=$ $\{l, \sigma, j, s, \lambda\}, g_{\alpha \alpha^{\prime}}^{( \pm)}$and $g_{\alpha \alpha^{\prime}}^{(i k)}$ are representatives of the operators $2 P^{ \pm}$and $2 P_{i k}$ in the MGL basis (Ref. $[6])$.

\section{2 s-wave approach. Elastic scattering}

In the $s$-wave approach there exists a single equation in the spin-quartet case for quantum state $\alpha=$ $\{0,1,1,3 / 2,0\}$ and our new results for $\mathrm{n}-\mathrm{d}$ and $\mathrm{p}$-d elastic amplitudes at $\mathrm{E}_{l a b}=14.1 \mathrm{MeV}$ calculated with the Malfliet-Tjon MT-I-III potential do not practically differ from our predictions (Ref. [1]) and those of the Los-Alamos/Iowa group (Ref. [2]) and we do not present them here. However in the spin-doublet case there exist three equations for quantum states $\alpha_{1}=\{0,0,0,1 / 2,0\}$ and $\alpha_{2}=$ $\{0,1,1,1 / 2,0\}$, one for $\Phi_{1, \alpha_{1}}$ and two for $\Phi_{2, \alpha_{i}},(i=1,2)$. For the ppn system, a set of equations is writen as:

$$
\begin{gathered}
{\left[E+\frac{\hbar^{2}}{2 m}\left(\partial_{x_{1}}^{2}+\partial_{y_{1}}^{2}\right)-v_{q}^{00}\right] \Phi_{1, \alpha_{1}}\left(x_{1}, y_{1}\right)=\left[v^{c}\left(x_{1}\right)+v_{p p}\left(x_{1}\right)\right]_{\alpha_{1} \alpha_{1}}\left[\Phi_{1, \alpha_{1}}\left(x_{1}, y_{1}\right)+\frac{1}{2} \int_{-1}^{1} d u h_{0000}^{0}\left(y_{1} / x_{1}, u\right)\right.} \\
\left.\times\left[\left(-\frac{1}{2} \Phi_{2, \alpha_{1}}\left(x_{2}, y_{2}\right)-\frac{1}{2} \Phi_{2, \alpha_{1}}\left(x_{3}, y_{3}\right)\right)+\left(\frac{\sqrt{3}}{2} \Phi_{2, \alpha_{2}}\left(x_{2}, y_{2}\right)+\frac{\sqrt{3}}{2} \Phi_{2, \alpha_{2}}\left(x_{3}, y_{3}\right)\right)\right]\right] \\
{\left[E+\frac{\hbar^{2}}{2 m}\left(\partial_{x_{2}}^{2}+\partial_{y_{2}}^{2}\right)-v_{q}^{00}\right] \Phi_{2, \alpha_{1}}\left(x_{2}, y_{2}\right)=\left[v_{p n}\left(x_{2}\right)\right]_{\alpha_{1} \alpha_{1}}\left[\Phi_{2 \alpha_{1}}\left(x_{2}, y_{2}\right)+\frac{1}{2} \int_{-1}^{1} d u h_{0000}^{0}\left(y_{2} / x_{2}, u\right)\right.} \\
\left.\left(-\frac{1}{2} \Phi_{1, \alpha_{1}}\left(x_{1}, y_{1}\right)-\frac{1}{2} \Phi_{2, \alpha_{1}}\left(x_{3}, y_{3}\right)-\frac{\sqrt{3}}{2} \Phi_{2, \alpha_{2}}\left(x_{3}, y_{3}\right)\right)\right] \\
\left.+\frac{1}{2} \int_{-1}^{1} d u h_{0000}^{0}\left(y_{2} / x_{2}, u\right)\left(\frac{\hbar^{2}}{2 m}\left(\partial_{x_{2}}^{2}+\partial_{y_{2}}^{2}\right)-\Phi_{q}^{00}\right] \Phi_{2, \alpha_{2}}\left(x_{2}, y_{2}\right)=\left[v_{p n}\left(x_{2}\right)\right]_{\alpha_{2} \alpha_{2}}\left[\Phi_{2 \alpha_{2}}\left(x_{2}, y_{2}\right)-\frac{\sqrt{3}}{2} \Phi_{2, \alpha_{1}}\left(x_{3}, y_{3}\right)+\frac{1}{2} \Phi_{2, \alpha_{2}}\left(x_{3}, y_{3}\right)\right)\right]
\end{gathered}
$$


In the s-wave approach the functions $g_{\alpha \alpha^{\prime}}^{( \pm)}$and $g_{\alpha \alpha^{\prime}}^{(i k)}$ are reduced to functions $h_{0000}^{0}\left(y_{i} / x_{i}, u\right)=$ $x_{i} y_{i} /\left(x_{k} y_{k}\right)$. Here $\left(x_{k}, y_{k}\right)$ are the coordinates of the integrand components $\Phi_{1,2}$ and $(k \neq i)$.

In the deuteron domain ( $x_{2}$ finite, $y_{2} \rightarrow \infty$ ) the asymptotic condition for the component $\Phi_{2}$ corresponding to elastic channel:

$$
\Phi_{2, \alpha_{2}}^{0,1 / 2,1 / 2}\left(x_{2}, y_{2}\right) \sim\left\{\delta_{\sigma 1} \delta_{J 1} e^{i \Delta_{0}^{c}} F_{0}^{c}\left(q y_{2}\right)+e^{-i \Delta_{0}^{c}}\left(G_{0}^{c}\left(q y_{2}\right)+i F_{0}^{c}\left(q y_{2}\right)\right) a_{01 / 2,01 / 2}^{1 / 2}\right\} \psi_{l}\left(x_{2}\right) .
$$

In formula (9) $F_{0}\left(q y_{2}\right)$ and $G_{0}\left(q y_{2}\right)$ are the regular and irreqular Coulomb functions and $\psi_{l}\left(x_{2}\right)$ is the $s$ - wave component of the deuteron wave function $(l=0)$. Amplitudes $\Phi_{1, \alpha_{1}}$ and $\Phi_{2, \alpha_{1}}$ have zero initial conditions and zero elastic asymptotics.

In the breakup domain we have for $\Phi_{1, \alpha_{1}}$ and $\Phi_{2, \alpha_{1,2}}$ the asymptotics:

$$
\Phi_{1, \alpha_{1}}^{0 \frac{1}{2} \frac{1}{2}} \sim e^{-W_{1}\left(\theta_{1}\right)} A_{1, \alpha_{1}}^{0 \frac{1}{2} \frac{1}{2}}\left(\theta_{1}\right), \quad \Phi_{2, \alpha_{1,2}}^{0 \frac{1}{2} \frac{1}{2}} \sim \mathcal{E}_{0} A_{2, \alpha_{1,2}}^{0 \frac{1}{2} \frac{1}{2}}\left(\theta_{2}\right), \quad \mathcal{E}_{0}=2 \pi \int_{-1}^{1} d u e^{i W_{2}\left(\hat{\mathbf{x}}_{2}, \hat{\mathbf{y}}_{2}\right)}, \quad u=\cos \left(\hat{\mathbf{x}}_{2} \hat{\mathbf{y}}_{2}\right) .
$$

In these formulas the Coulomb distorted phases $W_{1}$ and $W_{2}$ are as following

$$
W_{1}(\theta)=-\frac{1}{2 \sqrt{E}} \frac{m e^{2}}{\hbar^{2}} \frac{\ln (2 \sqrt{E} X)}{\cos \theta}, \cos \theta=\frac{x_{1}}{X},
$$

and

$$
W_{2}\left(\hat{\mathbf{x}}_{2}, \hat{\mathbf{y}}_{2}\right)=-\frac{1}{2 \sqrt{E}} \frac{m e^{2}}{\hbar^{2}} \frac{X}{\left|\mathbf{x}_{\mathbf{2}} / 2+\sqrt{3} \mathbf{y}_{\mathbf{2}} / 2\right|} \ln (2 \sqrt{E} X), \quad X=\sqrt{x_{i}^{2}+y_{i}^{2}} .
$$

The two doublet amplitudes are given as follows

$$
\begin{aligned}
& \mathcal{A}_{\alpha_{1}}\left(\theta_{2}\right)=A_{2, \alpha_{1}}\left(\theta_{2}\right)+\frac{1}{2} \int_{-1}^{1} d u h_{0000}^{0}\left(y_{2} / x_{2}, u\right) \\
& \left(g_{11} A_{1, \alpha_{1}}\left(\theta_{1}\right)-g_{11}^{(12)} A_{2, \alpha_{1}}\left(\theta_{3}\right)-g_{12}^{(12)} A_{2, \alpha_{2}}\left(\theta_{3}\right)\right) \\
& \mathcal{A}_{\alpha_{2}}\left(\theta_{2}\right)=A_{2, \alpha_{2}}\left(\theta_{2}\right)+\frac{1}{2} \int_{-1}^{1} d u h_{0000}^{0}\left(y_{2} / x_{2}, u\right) \\
& \left(g_{21} A_{1, \alpha_{1}}\left(\theta_{1}\right)-g_{21}^{(12)} A_{2, \alpha_{1}}\left(\theta_{3}\right)-g_{22}^{(12)} A_{2, \alpha_{2}}\left(\theta_{3}\right)\right) .
\end{aligned}
$$

Equations (6 - 8) violate the isospin symmetry because of distinctions between $\mathrm{nn}, \mathrm{pp}$ and $\mathrm{np}$ forces. The charge asymmetry is obtained by allowing the strengths of the central $n n$ and $p p$ forces to be different from $n p$ one. We modify MT-I-III potentials to reproduce singlet $\mathrm{n}-\mathrm{n}$ and $\mathrm{p}$-p scattering data by scaling the $p n$ potential by factors 0.982 and 0.9745 for $n n$ and $p p$, respectively. While MTI-III uses for $a_{\mathrm{np}}, a_{\mathrm{nn}}$ and $a_{\mathrm{pp}}$ the value of scattering length is $-23.5 \mathrm{fm}$, we modify the potential to produce their scattering lenghts to agreement with experimental data (Ref. [10]).

The accuracy of this adjustment procedure is checked by calculating binding energies for ${ }^{3} \mathrm{H}$ and ${ }^{3} \mathrm{He}$ using MT-I-III potential and its modifications. Our new results and previous ones from Ref. [7] and Ref. [8] obtained in isospin formalism are given in table 1. Our new results calculated applying a new set of three Faddeev equations with modified MT-I-III NN potentials are in a good agreement with those from Ref. [7] and Ref. [8]. Results for our Coulomb energy $\Delta B_{c}$ and CSB energy $\Delta B(\mathrm{CBS})$ are given in table 1 .

In the $s$-wave approach the value of $\Delta B_{c}$ is $661 \mathrm{keV}$ slightly different from the result of 693 $\mathrm{keV}$ (Ref. [9]). Our result for the charge-symmetry breaking energy $\Delta B(\mathrm{CSB}$ ) is $61 \mathrm{keV}$ close to $71 \mathrm{keV}$ evaluated by Miller et al. [10]. Our new results for phase shifts and elasticities for n-d 
Table 1. $B\left({ }^{3} \mathrm{H}\right)$ and $B\left({ }^{3} \mathrm{He}\right.$ ) binding energies (in $\mathrm{MeV}$ ), the Coulomb energy $\Delta B_{c}$ (in keV), CSB effect for energy $\Delta B\left(\mathrm{CSB}\right.$ ) (in keV). The results of Ref. [7] (Ref. [8]) are given in brackets (square brackets). $m_{n}\left(m_{p}\right)$ is the mass of neutron (proton).

\begin{tabular}{cccccccc}
\hline & $m_{n}$ & $m_{p}$ & $B\left({ }^{3} \mathrm{H}\right)$ & $B\left({ }^{3} \mathrm{He}\right)$ & $\Delta B_{c}$ & $\Delta B(\mathrm{CSB})$ \\
\hline MT-I-III & & 939.0 & 939.0 & 8.545 & & & \\
& & - & - & $(8.535)$ & $(7.868)$ & & \\
\multirow{2}{*}{ Modified MT-I-III } & $\mathrm{nn}$ & - & - & {$[8.54]$} & {$[7.88]$} & & \\
& $\mathrm{pp}$ & - & - & 8.396 & 7.735 & 661 & \\
& & - & & 7.674 & - & 61 \\
\hline
\end{tabular}

Table 2. Spin-doublet case. $\mathrm{n}-\mathrm{d}$ and $\mathrm{p}-\mathrm{d}$ elastic shifts and inelasticities at $\mathrm{E}_{l a b}=14.1 \mathrm{MeV}$.

\begin{tabular}{cccc|ccc}
\hline & \multicolumn{3}{c|}{$\mathrm{n}-\mathrm{d}$} & \multicolumn{3}{c}{$\mathrm{p}-\mathrm{d}$} \\
\hline & MT-I-III & {$[1]$} & modified MT-I-III & MT-I-III & {$[1]$} & modified MT-I-III \\
\hline$\delta(\operatorname{deg})$ & 106.16 & 105.47 & 105.56 & 111.05 & 108.06 & 110.76 \\
$\eta$ & 0.4653 & 0.4649 & 0.4744 & 0.533 & 0.4929 & 0.536 \\
\hline
\end{tabular}

and $\mathrm{p}$-d breakup scattering at $\mathrm{E}_{l a b}=14.1 \mathrm{MeV}$ calculated assuming the neutrons and protons to be distinguishable particles are presented in table 2. One concludes that CSB is visible in phase shifts and inelasticities for neutron-deuteron and proton-deuteron scattering at $\mathrm{E}_{l a b}=14.1 \mathrm{MeV}$. However these results have been obtained applying partly artifficial procedure for constructing $s$-wave singlet $n n$ and $p p$ components of MT-I-III potential. Therefore we are currently extending our studies using the charge dependent AV18 NN potential.

\section{Acknowledgements}

This work is supported by the NSF (HRD-1345219).

\section{References}

[1] V.M. Suslov and B. Vlahovic, Phys. Rev. C 69 (2004) 044003.

[2] J.L. Friar, G.L. Payne, W. Glöckle, D. Hüber, and H. Witala, Phys. Rev. C 51 (1995) 2356.

[3] A.A. Kvitsinsky, Yu.A. Kuperin, S.P. Merkuriev, A.K. Motovilov, S.L. Yakovlev, EChaYa, 17 (1986) 267-317.

[4] H. Witala, W. Glöckle, and H. Kamada, Phys. Rev. C 43 (1991) 1619.

[5] V.M. Suslov, M.A. Braun, I. Filikhin, B. Vlahovic, I. Slaus, Few-Body Syst. (2011) 50; 267.

[6] S.P. Merkuriev, C. Gignoux, A. Laverne, Annals of Phisics 99, 30-71 (1976).

[7] J.L. Friar, B.F. Gibson, G.L. Payne, Phys. Rev. C 24 (1981) 2279.

[8] J. Bernabeu, V.M. Suslov, T.A. Strizh, S.I. Vinitsky, Hyperfine interaction 110/102 (1996) 391399

[9] S.A. Coon and R.C. Barrett, Phys. Rev. C 36 (1987) 2189.

[10] G.A. Miller and W.T.H. van Oers, arXiv:nucl-th/9409013 